\section{Mortalidade, anos potenciais de vida perdidos e incidência de acidentes de trabalho na Bahia, Brasil}

\author{
Mortality, years of life lost, and incidence of \\ occupational accidents in the State \\ of Bahia, Brazil
}

Vilma Souza Santana 1

José Bouzas Araújo-Filho 1

Marlene Silva 1

Paulo Rogério Albuquerque-Oliveira 2

Anadergh Barbosa-Branco ${ }^{3}$

Letícia Coelho da Costa Nobre 4
I Instituto de Saúde Coletiva, Universidade Federal da Bahia, Salvador, Brasil. 2 Secretaria Executiva, Ministério da Previdência Social, Brasília, Brasil.

${ }^{3}$ Departamento de Saúde Coletiva, Universidade de Brasília, Brasília, Brasil. 4 Fundação Jorge Duprat Figueiredo de Segurança e Medicina do Trabalho Salvador, Brasil.

Correspondência V. S. Santana Programa Integrado em Saúde Ambiental e do Trabalhador, Instituto de Saúde Coletiva, Universidade Federal da Bahia. Rua Augusto Vianna s/n, 2o andar, Campus Universitário do Canela, Salvador, $B A$ 40110-040, Brasil. vilma@ufba.br

\section{Abstract}

This study of occupational accidents presents estimates for mortality, years of potential life lost, and cumulative incidence of severe cases (over 15 workdays lost) in Bahia State, Brazil, 2000. A correction factor was produced by comparing different data sources. Data were taken from compensation claims in the National Social Security Unified Benefits System (SUB), death certificates from the Ministry of Health Mortality Information System, and national census. Occupational accident-related mortality was estimated as 0.79 per 100,000 workers using the Mortality Information System, but increased to 13.17 per 100,000 using the SUB database. Assuming the latter result for the entire workforce produced a correction factor of 16.67 for the Mortality Information System database. Years of potential life lost were 23,249, and the cumulative incidence of severe occupational accidents was $2.3 \%$. Occupational accidents are preventable, but still common in Brazil. Underreporting is widespread, and corrected statistics need to be published, thereby turning this neglected public health problem into a policy priority.

Occupational Accidents; Potential Years of Life Lost; Incidence

\section{Introdução}

Acidentes de trabalho são uma das principais causas ocupacionais de morte em todo o mundo. Em um estudo recente, estimou-se em 350 mil o número de mortes por acidentes de trabalho típicos no mundo 1 , enquanto que os coeficientes de mortalidade variaram de 5,89 x 100 mil nos países de economia de mercado estabelecida a 23,10 x 100 mil em países asiáticos, ficando em 13,50 x 100 mil nos países da América Latina. Esses dados não incluem acidentes de trajeto ${ }^{2}$. No Brasil, os dados da Previdência Social permitem estimar para o ano de 2003 um coeficiente de mortalidade de 11,40 x 100 mil entre os trabalhadores elegíveis para o recebimento de benefícios 3,16 vezes maior do que o estimado para o mesmo período na Inglaterra, de 0,70 x 100 mil 4 . Como acidentes de trabalho acometem mais comumente pessoas jovens, no início da vida laboral, esses agravos podem afetar grandemente a capacidade produtiva e econômica do país.

O impacto social e econômico de mortes prematuras pode ser estudado epidemiologicamente com estimativas dos anos potenciais de vida perdidos, que expressa, com base na idade esperada em que ocorreria o óbito, a quantidade de anos que se deixou de viver. Embora menos divulgados do que os coeficientes de mortalidade por doenças e agravos ocupacionais, revelam uma dimensão importante da carga dos riscos do trabalho sobre a população, ao incorporar na sua 
medida uma ponderação que se eleva de modo inversamente proporcional à idade da morte 5 . Bailer et al. 6, ao analisarem estatísticas do sistema de vigilância de mortes ocupacionais dos Estados Unidos, identificaram como atividades com maior número de anos de vida perdidos o trabalho com máquinas ou correntes elétricas, enquanto as causas mais comuns foram a violência intencional (homicídios), quedas e acidentes de transporte. No Brasil, em 1991, as causas externas em geral representaram 3.417.264 anos potenciais de vida perdidos 7 , e mais tarde, com dados de 2003, calculados em 2.714.140 8, mas não foram apresentadas estimativas específicas para acidentes de trabalho.

Em todo o mundo, estatísticas sobre acidentes do trabalho são subestimadas, o que é relatado em vários estudos ${ }^{2}$. De acordo com Driscoll et al. ${ }^{1}$, as razões para erros nas estimativas de morbidade e mortalidade por acidentes de trabalho podem ser localizadas na definição, identificação e no registro. A definição envolve dificuldades tanto na delimitação do que é o fator de risco ou causal, suas circunstâncias de ocorrência e relação com o trabalho, o desfecho e a relação entre um e outro, o que impõe claras dificuldades de reconhecimento pelos profissionais de saúde. Esse aspecto é um forte determinante dos limites encontrados na identificação, pois o nexo causal está implícito no processo de diagnóstico, que por sua vez se encontra subjacente no registro. Vale ressaltar que nem todos os casos reconhecidos e diagnósticos são registrados devido a implicações políticas, jurídicas, conflitos de interesses econômicos, estigma, ou mesmo a simples negligência de parte de empregadores, profissionais de saúde e até mesmo dos principais interessados, os trabalhadores 3,9,10. Nos Estados Unidos, o sub-registro de acidentes de trabalho foi estimado entre $33 \%$ e $69 \%$, maior entre os trabalhadores autônomos e funcionários públicos 9 .

No Brasil, a subestimação de mortalidade e morbidade vem sendo descrita em estudos conduzidos em diferentes regiões do país ${ }^{3}$. Para acidentes fatais de trabalho, o registro de maior número de casos foi consistentemente encontrado entre os Boletins de Ocorrência nos distritos policiais, enquanto a subenumeração variou de cerca de $90 \%$ a 27\% em São Paulo, onde foi implantado um sistema de melhoria das informações para mortes por causas externas. A incidência de acidentes de trabalho não-fatais pode ser subestimada em até $90 \%$, de acordo com resultados de estudos populacionais 3 . Com base em resultados da magnitude de sub-registros é possível se calcular medidas epidemiológicas corrigidas, embora se adotando alguns pressupostos. Neste estudo, estima-se a mortalidade por acidentes de trabalho, os anos potenciais de vida perdidos, e também a incidência dos acidentes graves (com 15 ou mais dias de afastamento do trabalho), com base em dados do Ministério da Previdência e Assistência Social (MPAS), na Bahia, relativos aos benefícios acidentários para acidentes de trabalho, concedidos em 2000.

\section{Métodos}

Este estudo foi conduzido com dados secundários de diferentes bases, como o Sistema de Informações sobre Mortalidade (SIM), que corresponde aos dados das Declarações de Óbito de todo o país, e disponibilizado na Internet por meio do Departamento de Informática do SUS (DATASUS; http://www.datasus.gov.br). Utilizam-se também dados do Sistema Único de Benefícios (SUB), do MPAS, que mantém o registro das concessões de benefícios, incluindo-se as pensões de familiares por morte do contribuinte. Nesses dois sistemas constam as causas de óbito codificadas pela Classificação Estatística Internacional de Doenças e Problemas Relacionados à Saúde - 10a Revisão (CID-10) 11, o que permite a identificação dos casos de óbito por acidentes de trabalho, selecionando os casos classificados no capítulo XX - "causas externas" da CID-10. O número de trabalhadores em risco para esses acidentes, definido pela população economicamente ativa ocupada (PEA ocupada), foi empregado como denominador para o cálculo dos coeficientes de mortalidade específica. Estimativas foram obtidas de dados do Instituto Brasileiro de Geografia e Estatística (IBGE, http:/ / www.ibge.gov.br) e do Cadastro Nacional de Informações Sociais (CNIS), do MPAS, de onde se obteve o número de trabalhadores formalmente contratados e vinculados ao SUB. Todos os dados empregados foram os que se referiam ao Estado da Bahia, ano 2000.

Calculou-se a mortalidade dividindo-se o número de óbitos por acidentes de trabalho pela população em risco de morrer por estes eventos, considerando-se o tipo de vinculação com a respectiva base de dados disponível. Os coeficientes de mortalidade para o total da população $\left(\mathrm{CM}-\mathrm{AT}_{\text {geral }}\right)$ foram estimados com base no SIM/IBGE, e para os trabalhadores contribuintes e pertencentes ao Regime Geral de Benefícios da Previdência Social (CM-AT $\mathrm{formal}_{\text {la }}$ ), os dados foram provenientes do SUB/CNIS-MPAS. Para se es-

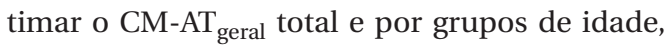
utilizou-se o número de óbitos com diagnósticos correspondentes a acidentes de trabalho, diagnóstico informado em um campo específico da Declaração de Óbito e registrados no SIM. O va- 
lor correspondente ao número da PEA ocupada foi obtido no IBGE. Todos os dados empregados correspondiam aos mesmos grupos de idade e ano de referência, para a Bahia.

Para se estimar a mortalidade com base nos dados da Previdência Social, empregaram-se os óbitos correspondentes aos despachos de pensões a familiares do falecido (B93), que infelizmente não contavam com o diagnóstico da causa de morte. Todavia, como a grande maioria das mortes relacionadas à ocupação é causada por acidentes de trabalho, considerou-se esta como a causa comum. Para estimar os anos potenciais de vida perdidos (APVP), consideraram-se as faixas etárias, para as quais os anos remanescentes de vida foram calculados subtraindo-se os anos de vida que seriam vividos caso o trabalhador não tivesse falecido (baseando-se na expectativa de vida para cada idade). Em seguida, multiplicaram-se esses valores pelo número de óbitos em cada faixa de idade, que foram então somados, de acordo com a fórmula abaixo, em que, $m_{i}$ é o número de mortes para a faixa etária $i$, e $a_{i}$ é a média da expectativa de vida na faixa etária $i$, sendo $i=1, \ldots, n$ no qual $n$ é o número de faixas de idade. Os valores correspondentes à expectativa de vida de cada grupo de idade foram definidos com a tabela empregada pelo MPAS 8 .

$$
A P V P_{i}=\sum m_{i} \times a_{i} \quad \text { (1) }
$$

Esses indicadores (CM-AT e APVP) foram calculados na forma bruta, ou seja, de acordo com os dados existentes nas bases de dados, e corrigidos para o sub-registro utilizando-se fatores de correção baseados em pressupostos construídos a partir de resultados de pesquisas realizadas com grandes amostras populacionais. Como os resultados de vários estudos, consistentemente, têm demonstrado que a base da Previdência Social capta mais casos do que o SIM, para trabalhadores formais 3 , utiliza-se a base do SUB como referência para se corrigir os resultados obtidos com o SIM. Assim, o fator de correção corresponde à razão entre a mortalidade estimada com dados da Previdência Social e do SIM. Além disso, como se atribui o mesmo coeficiente de mortalidade obtido para trabalhadores formais, para os não-formais assume-se como outro pressuposto que não existe diferença na mortalidade por acidentes de trabalho entre estes dois grupos de trabalhadores.

Para o cálculo da incidência cumulativa anual de acidentes de trabalho dividiu-se o número de casos de acidentes de trabalho não-fatais pelo número de trabalhadores em risco, para a população total $\left(\mathrm{IC}_{\text {geral }}\right)$ e específica para trabalhadores com contrato formal pertencentes ao SUB/ MPAS ( $\left(\mathrm{C}_{\mathrm{formal}}\right)$. Para as estimativas corrigidas para subenumeração, empregaram-se os resul- tados de um estudo de base populacional realizado na cidade de Salvador, no mesmo ano ${ }^{12}$, assumindo-se que a incidência cumulativa anual e a distribuição por idade da pesquisa eram semelhantes às de todo o Estado da Bahia. Aplicou-se a distribuição por idade da incidência a cada uma das faixas de idade da PEA ocupada, obtendo-se o número de casos esperados, para assim estimar o número total de acidentes de trabalho esperado. Notar que os dados foram restritos aos acidentes de trabalho graves, ou seja, com 15 ou mais dias perdidos de trabalho, para tornar compatível com o critério de concessão de benefícios. Para estimar a incidência de acidentes graves, os dados dos casos vieram do SUB, e os denominadores foram constituídos da média mensal de vínculos de contrato de trabalho constantes da base de dados do MPAS/ SUB. Para descrever a distribuição do não registro de Comunicação de Acidente de Trabalho (CAT) na base de dados dos benefícios pagos pela Previdência, foi estimada a proporção de benefícios concedidos sem este documento, de acordo com o tipo de benefício recebido pelo trabalhador.

Testes estatísticos não foram realizados por serem dados censitários para toda a população, e não se tratar de teste de hipóteses. O protocolo de pesquisa não foi submetido ao Comitê de Ética devido à natureza pública e administrativa dos dados, que não incluíam identificação do trabalhador ou da empresa.

\section{Resultados}

De acordo com dados do IBGE, a população economicamente ativa ocupada na Bahia, no ano de 2000, era de 4.581.593 indivíduos. Identificaram-se 36 óbitos na base do SIM com o diagnóstico de acidentes de trabalho como causa básica, ficando o coeficiente de mortalidade geral estimado (CM-AT geral $_{\text {) }}$ de 0,79/100 mil (Tabela 1 ). Trabalhadores de 35 a 39 anos apresentaram o maior risco de morte por esse agravo ocupacional, mais que o dobro do coeficiente global $\left(\mathrm{CM}-\mathrm{AT}_{\text {geral }}=1,65 / 100 \mathrm{mil}\right) . \mathrm{O}$ impacto na produtividade, estimado com o número de anos potenciais perdidos de vida, foi maior entre os indivíduos nas idades de 35 a 39 anos (333 APVP, 24\% do total APVP), embora apenas levemente maior do que no grupo entre 20-25 anos que contribuiu com 300 APVP (22\%). Em contraste, os óbitos por acidentes de trabalho registrados entre os contribuintes do MPAS, que corresponde a apenas uma parte da população geral, foram muito mais elevados ( $n=147)$. Considerando-se a população de trabalhadores com contrato formal de traba- 
Mortalidade por acidentes de trabalho e anos potenciais de vida perdidos (APVP) para o total da população ocupada, por faixa de idade. Estado da Bahia, Brasil, 2000

\begin{tabular}{|c|c|c|c|c|}
\hline $\begin{array}{l}\text { Faixa etária } \\
\text { (anos) }\end{array}$ & $\begin{array}{l}\text { Óbitos por } \\
\text { acidentes de trabalho * }\end{array}$ & $\begin{array}{l}\text { População } \\
\text { ocupada ** }\end{array}$ & $\begin{array}{l}\text { CM-ATgeral } \\
\text { (x } 100 \mathrm{mil})\end{array}$ & APVP $* \star \star *$ \\
\hline $10-17$ & 1 & 362.586 & 0,28 & 58 \\
\hline $18-19$ & 0 & 241.634 & - & - \\
\hline $20-24$ & 6 & 694.192 & 0,86 & 300 \\
\hline $25-29$ & 6 & 625.320 & 0,96 & 276 \\
\hline $30-34$ & 3 & 594.007 & 0,51 & 123 \\
\hline $35-39$ & 9 & 545.659 & 1,65 & 333 \\
\hline $40-44$ & 5 & 459.959 & 1,09 & 160 \\
\hline $45-49$ & 2 & 353.110 & 0,57 & 56 \\
\hline 50-59 & 2 & 458.356 & 0,44 & 44 \\
\hline $60-69$ & 2 & 183.124 & 1,09 & 30 \\
\hline$>70$ & 0 & 63.646 & - & - \\
\hline Total & 36 & 4.581 .593 & 0,79 & 1.380 \\
\hline \multicolumn{5}{|c|}{ CM-AT ${ }_{\text {geral }}:$ coeficientes de mortalidade para o total da população. } \\
\hline \multicolumn{5}{|c|}{ * Dados do Sistema de Informações sobre Mortalidade (SIM; http://www.datasus.gov.br); } \\
\hline \multicolumn{5}{|c|}{ ** Dados do Instituto Brasileiro de Geografia de Estatística (IBGE; http://www.ibge.gov.br); } \\
\hline
\end{tabular}

lho de 1.116.358, estima-se em 13,17/100 mil o coeficiente de mortalidade anual (Tabela 2). Com esses dados, e considerando-se que a distribuição dos óbitos por faixa de idade dos benefícios do MPAS era semelhante à da população geral, foi possível calcular os APVP na população previdenciária, estimada em 5.635 anos em 2000. Baseando-se no fator de correção, que foi calculado dividindo-se o coeficiente de mortalidade com base na MPAS/SUB pelo do SIM, 13,17/100 mil por 0,79/100 mil, obteve-se o fator de correção da taxa de mortalidade de 16,67. Conseqüentemente, o número de óbitos por acidentes de trabalho esperado na população geral seria de 603, o valor que deveria constar no SIM (Tabela 2). Com esses mesmos pressupostos, o APVP esperado aumentaria para 23.152 anos. Isso corresponde a um sub-registro de 94\% [1-(36/603) dos acidentes de trabalho fatais nesse ano no Estado da Bahia]. Na Tabela 3, apresenta-se a distribuição estimada dos 603 óbitos por acidentes de trabalho esperados no Estado da Bahia, por faixa de idade. Considerando-se a distribuição respectiva da PEA ocupada, observa-se que o coeficiente de mortalidade por idade variou entre $7,30 / 100$ mil a 27,60/100 mil, no grupo de 35 a 39 anos de idade. O número de APVP chega a 23.249 no total, que tende a se reduzir a partir dos 45 anos, embora se eleve na faixa de mais idade.
Para os acidentes de trabalho não-fatais, considerou-se apenas os que tinham 15 ou mais dias perdidos de trabalho, estimando-se o coeficiente de incidência cumulativa de acidentes de trabalho graves em $2,3 \%$ para a população ocupada em geral. Aplicando-se as estimativas por faixa de idade, para o total da PEA ocupada no estado, por idade, calcula-se em 104.884 o total de acidentes de trabalho graves (Tabela 4). Os mais velhos, entre 60 e 69 anos de idade, apresentaram o maior risco de acidentes de trabalho graves, $3,95 \%$, muito próximo ao estimado entre os jovens de 25 a 29 anos ( $\mathrm{IC}_{\text {geral }} 3,3 \%$ ).

Do total de benefícios da série 90 e, portanto, para agravos à saúde relacionados ao trabalho, concedidos na Bahia (2.857) em 2000, 61,67\% não tiveram CAT emitida. Nota-se que a maior proporção de CAT não emitida (96,7\%) refere-se aos benefícios da aposentadoria por invalidez (B92) e 91,35\% para o auxílio acidente (B94). Proporções menores de não emissão de CAT foram observadas para o benefício por auxílio doença por acidentes de trabalho ou incapacidade temporária (B91), que teve $56,02 \%$ de não registro, como pode ser observado na Tabela 5 . 
Mortalidade por acidentes de trabalho e anos potenciais de vida perdidos (APVP) corrigidos para a subenumeração dos óbitos. Estado da Bahia, Brasil, 2000.

\begin{tabular}{|c|c|c|c|c|c|}
\hline $\begin{array}{l}\text { Especificação da } \\
\text { população de referência }\end{array}$ & Indivíduos & $\begin{array}{l}\text { Óbitos } \\
\text { observados }\end{array}$ & $\begin{array}{l}\text { Coeficiente de } \\
\text { mortalidade } \\
\text { (x } 100 \mathrm{mil})\end{array}$ & $\begin{array}{l}\text { Número } \\
\text { corrigido } \\
\text { de óbitos }\end{array}$ & APVP \\
\hline População de trabalhadores previdenciários & 1.116 .358 * & 147 ** & 13,17 & --- & $5.635 * \star \star$ \\
\hline População economicamente ativa ocupada & $4.581 .593 \#$ & 36 & 0,79 & $603 \# \#$ & $23.152 \# \# \#$ \\
\hline
\end{tabular}

* Número de contribuintes para o Regime Geral de Benefícios da Previdência Social (Cadastro Nacional de Informações Sociais/Ministério da Previdência e Assistência Social). Dados do Ministério da Previdência e Assistência Social 8;

** Fonte: Sistema Único de Benefícios (SUB);

*** Assumindo-se a distribuição de idade observada com os dados do Sistema de Informações sobre Mortalidade (SIM), e a expectativa de vida adotada na Empresa de Tecnologia e Informações da Previdência Social (DATAPREV; http://www.previdencia.gov.br/pg_secundarias/beneficios_04_06-A.asp, acessado em 05/Mai/2005);

\# Dados do Instituto Brasileiro de Geografia e Estatística (IBGE; http://www.ibge.gov.br);

\#\# Assumiu-se que a mortalidade da população de trabalhadores previdenciários (13,2/100 mil trabalhadores) é igual à da população economicamente ativa ocupada. Assim, o fator de correção foi calculado pela razão entre a mortalidade estimada com os dados da Previdência Social e a estimada com os dados do SIM/IBGE para a população economicamente ativa ocupada - PEA ocupada $(13,2 / 0,79=16,7)$;

\#\#\# APVP corrigido.

Tabela 3

Mortalidade por acidentes de trabalho e anos potenciais de vida perdidos (APVP) corrigidos para a subenumeração dos óbitos, considerando-se o total da população economicamente ativa ocupada (PEA ocupada), por idade. Estado da Bahia, Brasil, 2000.

\begin{tabular}{|c|c|c|c|c|}
\hline $\begin{array}{l}\text { Faixa etária } \\
\text { (anos) }\end{array}$ & $\begin{array}{l}\text { Número corrigido de óbitos } \\
\text { por acidentes de trabalho * }\end{array}$ & $\begin{array}{c}\text { PEA } \\
\text { ocupada ** }\end{array}$ & $\begin{array}{l}\text { Mortalidade por acidentes de } \\
\text { trabalho corrigida (x } 100 \text { mil) }\end{array}$ & $\begin{array}{l}\text { APVP } \\
\text { corrigidos }\end{array}$ \\
\hline $10-17$ & 16,75 & 362.586 & 4,6 & 971,5 \\
\hline $18-19$ & 0,00 & 241.634 & - & - \\
\hline $20-24$ & 100,50 & 694.192 & 14,5 & $5.025,0$ \\
\hline $25-29$ & 100,50 & 625.320 & 16,1 & $4.623,0$ \\
\hline $30-34$ & 50,25 & 594.007 & 8,5 & $2.060,3$ \\
\hline $35-39$ & 150,75 & 545.659 & 27,6 & $5.577,8$ \\
\hline $40-44$ & 83,75 & 459.959 & 18,2 & $2.680,0$ \\
\hline $45-49$ & 33,50 & 353.110 & 9,5 & $1.072,0$ \\
\hline $50-59$ & 33,50 & 458.356 & 7,3 & 737,0 \\
\hline $60-69$ & 33,50 & 183.124 & 18,3 & 502,5 \\
\hline$>70$ & 0,00 & 63.646 & - & - \\
\hline Total & 603,00 & 4.581 .593 & 13,2 & $23.249,1$ \\
\hline
\end{tabular}

* Assumiu-se que a mortalidade da população de trabalhadores previdenciários (13,2/100 mil habitantes) é igual à da população economicamente ativa ocupada. Assim, o fator de correção foi calculado pela razão entre a mortalidade estimada com os dados da Previdência Social e a estimada com os dados do Sistema de Informações sobre Mortalidade/Instituto Brasileiro de Geografia e Estatística (SIM/IBGE) para a PEA ocupada (13,2/0,79 = 16,7);

** Dados do IBGE (http://www.ibge.gov.br).

\section{Discussão}

Os resultados deste estudo mostram a elevada carga dos acidentes de trabalho para a população, na Bahia, em 2000, conforme revelada pela mortalidade, anos perdidos de vida e incidên- cia cumulativa dos acidentes não-fatais. Devido ao expressivo sub-registro de casos identificado, constatou-se que não é possível considerar apenas achados brutos, sem correções que permitam o conhecimento de estimativas mais acuradas. Revela-se também que o sub-registro de 
Incidência de acidentes de trabalho graves e o número de acidentes de trabalho estimado corrigindo-se para a subenumeração, considerando-se o total da população economicamente ativa ocupada (PEA ocupada), por idade. Salvador, Estado da Bahia, Brasil, 2000

\begin{tabular}{lccc}
\hline $\begin{array}{l}\text { Faixa etária } \\
\text { (anos) }\end{array}$ & $\begin{array}{l}\text { Incidência de acidentes de } \\
\text { trabalho em Salvador (\%)* }\end{array}$ & $\begin{array}{c}\text { PEA } \\
\text { ocupada ** }\end{array}$ & $\begin{array}{c}\text { Número estimado corrigido } \\
\text { de acidentes graves *** }\end{array}$ \\
\hline $10-17$ & 1,69 & 362.586 & 6.128 \\
$18-19$ & 2,70 & 241.634 & 6.524 \\
$20-24$ & 2,44 & 694.192 & 16.938 \\
$25-29$ & 3,30 & 625.320 & 20.636 \\
$30-34$ & 2,47 & 594.007 & 14.672 \\
$35-39$ & 1,08 & 545.659 & 5.893 \\
$40-44$ & 2,88 & 459.959 & 13.247 \\
$45-49$ & 1,96 & 353.110 & 6.921 \\
$50-59$ & 1,46 & 458.356 & 6.692 \\
$60-69$ & 3,95 & 183.124 & 7.233 \\
$>70$ & - & 63.646 & - \\
Total & 2,30 & 4.581 .593 & 104.884
\end{tabular}

* Incidência cumulativa de acidentes graves em uma amostra da população economicamente ativa de Salvador. Fonte: Santana et al. 12. Não se empregaram dados do Ministério da Previdência e Assistência Social (MPAS) por faltarem informações sobre idade;

** Dados do Instituto Brasileiro de Geografia de Estatística (IBGE; http://www.ibge.gov.br);

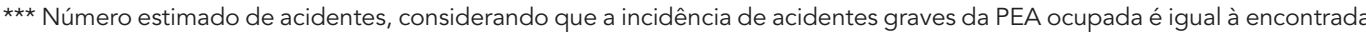
em estudo de base populacional na Região Metropolitana de Salvador 12.

Proporção de benefícios acidentários da Previdência sem Comunicação de Acidente de Trabalho (CAT), por tipo de benefício. Estado da Bahia, Brasil, 2000.

\begin{tabular}{|c|c|c|c|}
\hline Tipo de benefício acidentário & Benefícios concedidos & Benefícios sem CAT & $\%$ \\
\hline B91 - auxílio doença por acidente do trabalho com incapacidade temporária & 2.333 & 1.307 & 56,0 \\
\hline B92 - aposentadoria por invalidez por acidente do trabalho & 273 & 264 & 96,7 \\
\hline B93 - pensão por morte por acidente do trabalho & 147 & 96 & 65,3 \\
\hline B94 - auxílio acidente & 104 & 95 & 91,4 \\
\hline Total & 2.857 & 1.762 & 61,7 \\
\hline
\end{tabular}

Fonte: Ministério da Previdência Social (http://www.previdencia.gov.br/aeps2005/14_01_01_01.asp, acessado em 26/Ago/2003).

acidentes de trabalho é bem pior na base SIM em comparação com a da Previdência. Por exemplo, o coeficiente de mortalidade por acidentes de trabalho com base nos dados registrados no SIM, para o total da PEA ocupada foi 0,79/100 mil, muito menor do que o estimado com dados dos benefícios previdenciários, cerca de 13,17/100 mil. Isso representa uma diferença de 16,67 vezes, embora as populações de referência sejam distintas, trabalhadores em geral e formais, respectivamente, sendo estes últimos pertencentes ao SUB do Instituto Nacional de Seguridade So- cial (INSS). O número de anos perdidos de vida chegou a 23.152 anos, um valor expressivo e que indica a carga dos acidentes de trabalho entre os trabalhadores mais jovens, que além de uma perda irreparável para familiares, impacta negativamente sobre o desenvolvimento do país. Os acidentes de trabalho não-fatais acometem um grande número de trabalhadores, tendo sido estimado 104.884 casos em todo o estado por ano, limitando-se este número aos casos considerados graves por terem produzido afastamento do trabalho por pelo menos 15 dias. Esses casos 
também se concentram entre os trabalhadores adultos jovens de 20 a 35 anos, embora a maior incidência cumulativa tenha sido estimada na categoria de idade mais avançada.

Os resultados, além de demonstrarem o subregistro dos casos tanto fatais quanto não-fatais, permitem um melhor delineamento da realidade ao apresentar medidas corrigidas. Apesar dos dados da Previdência serem melhores do que os do SIM, verificou-se também que acidentes e doenças do trabalho de grande parte dos trabalhadores do SUB são reconhecidos sem o documento de registro formal que é a CAT. Verificou-se que esse documento é ainda insuficientemente empregado, com um grande porcentual de benefícios previdenciários por acidentes de trabalho por causas externas sendo gerados sem a CAT. Esses casos recebem o diagnóstico com nexo causal ocupacional, apenas durante a perícia médica, para patologias supostamente comuns à época da entrada do trabalhador no sistema.

A grande carga de acidentes de trabalho identificada neste estudo na Bahia, independentemente das suas causas específicas, revela, para além dos números, a sua dimensão trágica, invisível e de injustiça: são mortes e traumas evitáveis, pois são decorrentes de atividades para a produção, ou seja, tarefas realizadas de natureza intencional. Esse cenário é muito próximo do estimado para todo o país 3 e outros países da América Latina e Caribe ${ }^{2,13}$. Todavia, é muito maior do que as estimativas de países onde é notória uma tradição de políticas públicas avançadas e efetivas dirigidas à promoção da saúde e segurança dos trabalhadores, como a Dinamarca, com mortalidade por acidentes de trabalho de apenas 2,90 x 100 mil 2. Estudos sobre a incidência de acidentes de trabalho não-fatais são menos comuns devido a limitações nas estatísticas disponíveis. $\mathrm{Na}$ Inglaterra em 2000, a incidência de acidentes de trabalho registrada foi de $0,587 \%$ trabalhadores, e de $1,33 \%$ para todos os casos potencialmente registráveis 4, dados muito abaixo dos encontrados neste estudo. Todavia, as diferenças são menores do que as observadas para a mortalidade. Esse achado pode ser evidência de maior sub-registro para casos menos graves, mas não existem dados que permitam conclusões seguras, um aspecto importante a ser considerado em estudos futuros. Não foram encontrados estudos que tenham estimado anos perdidos de vida por acidentes de trabalho no país, de modo a se poder comparar com os resultados desta pesquisa.

Entre as razões para diferenças na carga de acidentes de trabalho entre países da América Latina e regiões com indicadores mais favoráveis, um estudo apontou as diferenças na estrutura do mercado de trabalho, o perfil produtivo de- marcando diferenças na concentração de riscos, a existência de grupos vulneráveis, como os de baixa escolaridade e jovens, e a disponibilidade de recursos como sistemas de saúde do trabalhador ou sindicatos, por exemplo ${ }^{13}$. Todavia, estudos brasileiros têm mostrado que inexistem diferenças significativas no risco de acidentes de trabalho não-fatais ao se comparar trabalhadores formais e informais 14, uma categorização importante no mercado de trabalho do Brasil. E em relação ao perfil produtivo, quando se limita a comparação a um mesmo ramo de atividade como o da construção, nota-se que ainda assim persistem expressivas diferenças na mortalidade por acidentes de trabalho quando se contrasta com estimativas de países ricos 15 . É difícil imaginar que diferenças entre países sejam devidas a aspectos individuais relativos aos trabalhadores, sendo mais plausível apontar aspectos das políticas de proteção ao trabalhador, tanto em sua formulação, organização, quanto efetivação, em suas variadas dimensões, sejam técnicas, sociais, econômicas, culturais ou políticas. A má qualidade dos programas de controle de riscos e saúde dos trabalhadores foi demonstrada com dados para esse mesmo estado em estudo recente 16 . Não foram encontrados estudos que tenham focalizado avaliações de programas de intervenção ou políticas de proteção da saúde e segurança de trabalhadores, seja em empresas ou regiões administrativas no Brasil ou em outros países. São também muito raros os estudos que buscam uma melhor compreensão das diferenças entre os diversos países, que com seus diferentes cenários relativos à prevenção de acidentes ocupacionais, poderiam contribuir para o aprimoramento das políticas e modelos de atuação pertinentes.

O grande sub-registro de óbitos por acidentes de trabalho no sistema SIM não é surpreendentemente considerando os resultados dos estudos que focalizaram a qualidade das fontes de dados, que evidenciaram sub-registro entre $39 \%$ e $95 \%$, conforme a revisão feita por Santana et al. ${ }^{3}$. Muitos acidentes fatais deixam de ser reconhecidos como causados por fatores ocupacionais por não ser de rotina a inclusão de perguntas sobre as circunstâncias da ocorrência durante a obtenção de informações sobre o acidente 17,18. Os dados deste estudo também revelam que embora o número de óbitos identificados no SIM seja menor que o encontrado na base do MPAS, esta também parece estar afetada pela subenumeração, considerando a elevada porcentagem de benefícios sem a emissão de CAT, tradicionalmente empregada para a computação de estatísticas oficiais brasileiras sobre doenças e acidentes de trabalho 2 . Estudos que compararam dados de inquérito da po- 
pulação geral, de base comunitária, empregando entrevistas domiciliares com os estimados com dados da Previdência baseados no registro da CAT, encontraram sub-registro de acidentes de trabalho não-fatais em relação ao SIM de $54,1 \%$ e de 22,4\% em relação à Previdência 19; e de $57,9 \%$ com dados da população geral e de 28,9\% quando se utiliza a CAT 20. Em 2002, cinco anos depois, repetiu-se a pesquisa de Botucatu, Estado de São Paulo, empregando-se a mesma metodologia. Verificou-se que a subenumeração dos acidentes de trabalho com as CAT foi ainda maior do que a encontrada no estudo anterior, $79,5 \%$ de casos não registrados 21 , indicando que aparentemente a tendência é de piora da qualidade deste sistema. É também presumível que haja diferenças importantes nos índices de subregistro previdenciários entre as regiões do país, de acordo com as formas de gestão do trabalho das empresas, especialmente naquelas que utilizam a terceirização, que apresentam maior mortalidade e morbidade ocupacional 22 .

É surpreendente que seja mais da metade a porcentagem de benefícios ocupacionais concedidos sem a CAT, cujo nexo causal se baseou em evidências médicas ou fornecidas pelo trabalhador diretamente à Previdência. Assim, esse importante instrumento se revela com pequeno valor preditivo para acidentes de trabalho entre trabalhadores cobertos pela Previdência, e até mesmo para os benefícios por acidente de trabalho. Essa constatação é crucial para se pensar em modificações no sistema de registro de acidentes de trabalho para os trabalhadores do sistema SUB. É possível que estimativas por acidentes de trabalho baseadas nos registros do SUB se aproximem mais dos dados reais do que aqueles limitados à CAT (ou do que aquelas derivadas da base de dados das CAT), visto que são óbvias e amplamente documentadas as razões para a não emissão da CAT fora do sistema 3,23. No entanto, não se pode descartar a hipótese de que o total de acidentes de trabalho nessa base esteja superestimado, pois um único acidente pode gerar vários tipos de benefícios, como para os acidentes fatais. Presume-se que, antes da aposentadoria por invalidez ou da concessão do auxílio acidente, o trabalhador tenha recebido, por algum tempo, o auxílio doença acidentário, enquanto mantinha-se afastado do trabalho. Mesmo para este, que teoricamente seria o benefício de "porta de entrada" do sistema para os casos não-fatais, pode-se considerar bastante elevado o índice de não emissão de CAT encontrado neste estudo. Nota-se que não existem dados na literatura sobre esse fato, embora sejam vários os autores que discutem a qualidade dos dados sobre acidentes de trabalho no país 3 . Da- da a universalidade da atenção à saúde dos trabalhadores no âmbito do Sistema Único de Saúde (SUS), urge avançar na estruturação de rede integrada de informações em saúde do trabalhador que compatibilize e interligue as diversas bases de dados e sistemas de informação 23 .

Com os resultados desta pesquisa, pode-se concluir que é grande o impacto sobre a produção econômica dos acidentes de trabalho, fatais e não-fatais, considerando-se o número elevado e os anos potenciais perdidos de vida, no Estado da Bahia, em 2000. Essa carga afeta principalmente os indivíduos jovens, que contribuem com a grande parte dos óbitos por acidentes de trabalho, da morbidade, incapacidade permanente ou invalidez, e conseqüentemente para os custos diretos e indiretos. Infelizmente não foi possível estimar os anos perdidos por morte e/ou qualidade de vida, o DALY (Disability Adjusted Life Years Lost), porque não se dispunha, na base de dados da Previdência (SUB), dos dados necessários. Um dos problemas observados em uma das fontes de dados disponíveis foi o tipo de agregação de algumas variáveis como a idade. Outras bases disponibilizavam apenas totais, ora por ano, ora por estado, ou mesmo distribuídos por mês/região. As bases de dados de vínculos de trabalho não são consistentes e não apresentam um registro identificador, útil para se operar com o método de captura-recaptura. Isso se reflete tanto para a obtenção do número de acidentes de trabalho quanto para a composição de denominadores 24 , um problema que não é limitado ao Brasil.

Os resultados deste estudo, embora plausíveis e consistentes com achados de outras pesquisas no Brasil, devem ser analisados com cuidado, em especial o tratamento das correções das estimativas e a extrapolação a partir de resultados de pesquisas conduzidas com amostras para outras populações. Entretanto, é cada vez mais comum esse tipo de procedimento, em particular quando se tratam de estimativas de carga de doença, já que muitas patologias não são registradas universalmente de modo adequado, conforme apresentado nesta pesquisa. Importante é a constatação de que há mais de uma década vários estudos vêm revelando esse problema e muito pouco foi realizado no sentido de se delinear um quadro mais realístico da situação nacional relativa a esta questão. Não foi possível validar internamente os dados das bases empregadas, analisando-se os casos identificados individualmente, por ter sido omitida a identidade do trabalhador na base SUB, e como faltavam dados sobre incapacidade para a população geral não se estimou o DALY, outra importante medida de carga de doença. 
Neste estudo fica evidente a invisibilidade dos acidentes ocupacionais e a grande carga que representa na Bahia. Vários estudos têm apontado a expressiva carga de acidentes de trabalho no Brasil, mas poucos têm focalizado a situação em particular nos diversos estados, como neste estudo, o que pode contribuir para o estabelecimento de prioridades locais e estratégias específicas, potencialmente mais eficientes no controle deste importante problema de saúde pública. A Bahia dispõe de profissionais qualificados e recursos de infra-estrutura que estão sendo largamente ampliados com a implantação da Rede Nacional de Atenção Integral à Saúde do Trabalhador no SUS (RENAST) na área da saúde do empregado, mas esforços entre as diversas instituições que compõem o sistema de saúde e de proteção do trabalhador deverão se voltar para o enfrentamento do problema da qualidade dos dados disponíveis, ao lado da efetivação de medidas que resultem em redução da mortalidade e incidência de acidentes de trabalho. A necessidade de melhorias no sistema de informações de doenças e acidentes ocupacionais no país foi um importante e bastante discutido tema durante a III Conferência Nacional de Saúde, e algumas medidas como a Portaria $n^{\circ}$. 777/28, de abril de 2004, são evidências disto. Estudos futuros deverão enfatizar as incapacidades, empregando medidas como o DALY, para uma adequada avaliação da carga das patologias ocupacionais, bem como medidas epidemiológicas de impacto populacional potencial como o risco atribuível à ocupação e o número de casos evitáveis, importantes informações para a tomada de decisão no campo das políticas de saúde. Projetos de intervenção que implementem ações visando ao aumento do registro em locais estratégicos como os serviços de emergência, ou estudos qualitativos sobre as percepções dos profissionais de saúde, empregadores e empregados sobre a informação de doenças ocupacionais poderão repercutir positivamente no conhecimento e nas condições de saúde da população trabalhadora no Brasil.

\section{Resumo}

Neste estudo estimam-se a mortalidade por acidentes de trabalho, anos potenciais de vida perdidos, e também a incidência de acidentes de trabalho graves (mais de 15 dias de afastamento), na Bahia, Brasil, no ano 2000. Fatores de correção foram elaborados comparando-se diferentes fontes de dados. Foram empregados benefícios da Previdência Social do Sistema Único de Benefícios (SUB), do Sistema de Informações sobre Mortalidade (SIM) do Ministério da Saúde, e Censo Demográfico. A mortalidade por acidentes de trabalho foi de 0,79 100 mil trabalhadores, com base no SIM, mas com o SUB a estimativa é de 13,17 x $100 \mathrm{mil}$. Assumindo-se esta medida para todos os trabalhadores, estima-se um fator de correção para o SIM de 16,67. A estimativa de anos potenciais de vida perdidos foi de 23.249 e a incidência de acidentes de trabalho graves com pelo menos duas semanas de afastamento foi de 2,3\%. Acidentes de trabalho são evitáveis, mas ainda comuns no país. A subenumeração é expressiva, e estatísticas corrigidas deveriam ser estimadas e divulgadas contribuindo para a priorização desse negligenciado problema de saúde pública.

Acidentes de Trabalho; Anos Potenciais de Vida Perdidos; Incidência

\section{Colaboradores}

Todos os autores participaram da elaboração do artigo. V. S. Santana participou na concepção e elaboração do projeto, recrutamento da equipe, preparação e limpeza da base de dados, análise e redação do artigo. J. B. Araújo-Filho contribuiu na limpeza e preparação da base de dados, análise e redação do artigo. M. Silva colaborou na revisão da literatura, análise e redação do artigo. P. R. Albuquerque-Oliveira obteve os dados necessários para a composição da base de dados, e juntamente com A. Barbosa-Branco participou na preparação da base de dados, codificando, organizando variáveis e contribuindo na redação do artigo. L. C. C. Nobre colaborou na revisão da literatura, revisão geral da consistência dos resultados e redação.

\section{Agradecimentos}

À Área Técnica de Saúde do Trabalhador, da Secretaria de Ações Básicas, Ministério da Saúde, pelo financiamento (processo no. 3478/2001) e ao Conselho Nacional de Desenvolvimento Científico e Tecnológico (CNPq), pela bolsa de produtividade em pesquisa (processo $\mathrm{n}^{\circ}$. 522621/96-1). 


\section{Referências}

1. Driscoll T, Takala J, Steenland K, Corvalan C, Fingerhut M. Review of estimates of the global burden of injury and illness due to occupational exposures. Am J Ind Med 2005; 48:491-502.

2. Takala J. Global estimates of fatal occupational injuries. Epidemiology1999; 10:640-6.

3. Santana VS, Nobre L, Waldvogel B. Acidentes de trabalho no Brasil entre 1994 e 2004 - uma revisão. Ciênc Saúde Coletiva 2005; 10:841-55.

4. Health and Safety Commission. Health and safety statistics 2004/05. http://www.hse.gov.uk/ statistics/overall/hssh0405.pdf (acessado em 23/ Nov/2005).

5. Gardner JW, Sanborn JS. Years of potential life lost (YPLL) - what does it measure? Epidemiology 1990; 1:322-9.

6. Bailer AJ, Bena JF, Stayner LT, Halperin WE, Park RM, Diversity of trends in occupational injury mortality in the United States 1980-96. Inj Prev 2003; 9:9-14.

7. Iunes RP. III - Impacto econômico das causas externas no Brasil: um esforço de mensuração. Rev Saúde Pública 1997; 31(4 Suppl):38-46.

8. Ministério da Previdência e Assistência Social. Anuário estatístico da previdência social - 2004. http://www.previdenciasocial.gov.br/estatisticas (acessado em 05/Mai/2005).

9. Leigh JP, Marcin JP, Miller TR An estimate of the U.S. Government's undercount of nonfatal occupational injuries. J Occup Environ Med 2004; 46:10-8.

10. Schulte PA Characterizing the burden of occupational injury and disease. J Occup Environ Med $2005 ; 47: 607-22$

11. Organização Mundial da Saúde. Classificação estatística internacional de doenças e problemas relacionados à saúde, 10a revisão. v. 1. São Paulo: Centro Colaborador da OMS para a Classificação de Doenças em Português; 1995.

12. Santana VS, Maia AP, Carvalho C, Luz G. Acidentes de trabalho não fatais: diferenças de gênero e tipo de contrato de trabalho. Cad Saúde Pública 2003; 19:481-93.
13. Giuffrida A, Iunes RF, Savedoff WD. Economic and health effects of occupational hazards in Latin America and the Caribbean. Washington DC: Inter-American Development Bank; 2001. (Technical Series, SOC - XXX).

14. Santana VS, Loomis D. Informal jobs and nonfatal occupational injuries. Ann Occup Hyg 2004; 48:147-57.

15. Mital A, Pennathur A, Kansal A. Non-fatal occupational injuries in the United States. Part I - Overall trends and data summaries. International Journal of Industrial Ergonomics 1999; 25:109-29.

16. Miranda CR, Dias CR. PPRA/PCMSO: auditoria, inspeção do trabalho e controle social. Cad Saúde Pública 2004; 20:224-32.

17. Beraldo PSS, Medina MG, Borba EA, Silva LP. Mortalidade por acidentes do trabalho no Brasil: uma análise das declarações de óbito, 1979-1988. Inf Epidemiol SUS 1993; 1:41-54.

18. Hennington EA, Cordeiro R, Moreira Filho DC. Trabalho, violência e morte em Campinas, São Paulo, Brasil. Cad Saúde Pública 2004; 20:610-7.

19. Binder MCP, Cordeiro R. Sub-registro de acidentes do trabalho em localidade do Estado de São Paulo, 1997. Rev Saúde Pública 2003; 37:409-16.

20. Barata RCB, Ribeiro MCSA, Moraes JC. Acidentes de trabalho referidos por trabalhadores moradores em área urbana no interior do Estado de São Paulo em 1994. Epidemiol Serv Saúde 2000; 9:199-210.

21. Cordeiro R, Sakate M, Clemente APG, Diniz CS, Donalísio MR. Subnotificação de acidentes de trabalho não fatais em Botucatu, SP, 2002. Rev Saúde Pública 2005; 39:254-60.

22. Quinlam M, Mayhew C, Bohle P. The global expansion of precarious employment, work disorganization, and consequences for occupational health: a review of recent research. Int J Health Serv 2001; 31:335-413.

23. Facchini LA, Nobre LCC, Faria NMX, Fassa AG, Thumé E, Tomasi E, et al. Sistema de informação em saúde do trabalhador: desafios e perspectivas para o SUS. Ciênc Saúde Coletiva 2005; 10:857-67.

24. Binder MCP, Wludarski SL, Almeida IM. Estudo da evolução dos acidentes do trabalho registrados pela Previdência Social no período de 1995 a 1999, em Botucatu, São Paulo. Cad Saúde Pública 2001; 17:915-24.

Recebido em 03/Abr/2006

Versão final reapresentada em 06/Mar/2007

Aprovado em 19/Abr/2007 